\title{
A chemosystematic investigation of selected Stichococcus-like organisms (Trebouxiophyta)
}

\author{
Anh Tu Van*, Ulf Karsten and Karin Glaser \\ Department of Applied Ecology and Phycology, Institute of Biological Sciences, University of Rostock, Albert-Einstein- \\ Straße 3, 18059 Rostock, Germany
}

The taxonomy of green microalgae relies traditionally on morphological traits but has been rapidly changing since the advent of molecular methods. Stichococcus Nägeli is a cosmopolitan terrestrial algal genus of the class Trebouxiophyceae that has recently been split into seven lineages, which, along with Pseudostichococcus, comprise the Stichococcuslike group; there is a need to further characterize these genera, since they are morphologically enigmatic. Here we used organic osmolytes as chemotaxonomic marker to verify the phylogenetic position of Stichococcus-like strains and were also able to exclude a strain hitherto identified as Gloeotila contorta from this group. Stichococcus-like organisms, including those recently revised, were characterized by the production of the polyol sorbitol and the disaccharide sucrose in high amounts, as is typical of Prasiola-clade algae. The results demonstrate that organic osmolyte chemotaxonomy can support green algal taxonomic designations as fundamental research.

Key Words: aeroterrestrial algae; chemotaxonomy; osmolytes; Pseudostichococcus; sorbitol; Stichococcus; sucrose

\section{INTRODUCTION}

Aeroterrestrial algae are abundant and ubiquitous microalgae occurring on various substrates like soil, tree barks, or man-made surfaces. However, many algae remain enigmatic in terms of their taxonomic identities and thus true biodiversity (Guiry 2012). One group, the Stichococcus-like algae within the order Prasiolales (Trebouxiophyceae) are of particular importance in terrestrial habitats, as they are ubiquitous and abundant in terrestrial environments (Rindi 2007, Hodač et al. 2016) inhabiting diverse habitats such as tree bark (Handa et al. 2003, Neustupa and Škaloud 2008, 2010), surfaces of buildings (facades, roof-tiles, etc.) and sculptures (Uher 2008, Gustavs et al. 2011, Hallmann et al. 2013), rocks (Hallmann et al. 2013), soil (Hodač et al. 2016) and biological soilcrusts (Ferrenberg et al. 2017, Glaser et al.
2018, Sommer et al. 2020a).

Recent efforts to delineate morphologically simple green microalgae such as Stichococcus have relied on molecular phylogeny. However, truly multiphasic approaches using physiological data is hitherto underutilized, with comparatively few studies to date focusing on physiological or chemotaxonomic data to bolster molecular interpretations (Pollio et al. 1997, Neustupa et al. 2007, Darienko et al. 2010, Karsten et al. 2016). Although Stichococcus bacillaris is an important alga in bioeconomic applications especially for fatty acids in biofuel production, only limited knowledge exist on closely related Stichococcus-like organisms (Olivieri et al. 2011, Mutaf et al. 2019).

One aspect of biochemical traits is of particular inter-

\section{(ब) (1) $(3$}

This is an Open Access article distributed under the terms of the Creative Commons Attribution Non-Commercial License (http://creativecommons.org/licenses/by-nc/3.0/) which permits unrestricted non-commercial use, distribution, and reproduction in any medium, provided the original work is properly cited.
Received January 21, 2021, Accepted June 5, 2021

*Corresponding Author

E-mail: anh.van@uni-rostock.de

Tel: +49-381-498-6095, Fax: +49-381-498-6072 
est: the presence of low molecular weight carbohydrates (LMWC) has both consequences for the cell biology and physiology in terms of osmotic stress tolerance (Roberts 2005, Holzinger and Karsten 2013, Holzinger and Pichrtová 2016) and has been demonstrated to be a stable taxonomic character (Gustavs et al. 2011, Darienko et al. 2015, Hotter et al. 2018). It also provides valuable information on the ecophysiology of the strains, which is usually missing from classical phylogenetic analyses. This approach has been used to analyze carbohydrates as primary metabolites, secondary metabolites, and beyond, chemotaxonomically in both macro- and microalgae. For example, Eggert and Karsten (2010) have shown that the sugars floridoside is accumulated in most Rhodophyta, and digeneaside, a related compound, is instead a marker of specific families within the phylum (Karsten et al. 1999, 2007, Kamiya et al. 2016). The sunscreen and antioxidant compounds mycosporin-like amino acids, abundant in many algal groups (Oren and Gunde-Cimerman 2007), have been used as taxonomic markers in the genera Bostrychia (Ceramiales, Rhodophyta) (Orfanoudaki et al. 2020) and Klebsormidium (Streptophyta) (Kitzing et al. 2014) as well as in Prasiola and Watanabea (Trebouxiophyceae) (Karsten et al. 2005).

Prior research in the Prasiolales showed that Stichococcus have a signature of osmolytes (sorbitol, sucrose, and proline) in response to salt stress (Brown and Hellebust 1978, Hellebust 1985). Hotter et al. (2018) proposed sorbitol as a chemotaxonomic marker for the Prasiola-clade within the Trebouxiophyceae, even under non-saline growth conditions. However, other Stichococcus-like genera such as Pseudostichococcus have not been studied so far. Furthermore, the recent taxonomical revision of the Stichococcus-like algae split those into the genera Stichococcus, Protostichococcus, Deuterostichococcus, Tritostichococcus, and Tetratostichococcus (Pröschold and Darienko 2020). This necessitates further investigation, as these revisions affect organisms that are morphologically diverse at the taxon level, yet very closely related at the genus level.

In this study, a wide variety of Stichococcus and Stichococcus-like (sensu Nägeli; the latter expanded on by Pröschold and Darienko 2020) strains were examined for their ability to synthesize sorbitol, the main osmolyte marker of the Prasiola-clade, in order to improve the current knowledge of the physiology of these strains, as they have not yet been extensively characterized. We also investigated Pseudostichococcus, which, although distinct from Stichococcus and Stichococcus-like organisms, is morphologically and genetically similar enough to warrant a more precise characterization. A wide variety of LMWC (e.g., ribitol, arabitol, xylitol) was used as standards, beyond those typical of Prasiola-clade algae, as it was unknown what the expression profile of these strains would be. Finally, this method was performed on two strains putatively outside of this group in order to demonstrate its taxonomic utility.

\section{MATERIALS AND METHODS}

In total, 26 strains were used for chemotaxonomic analyses (Table 1). Established unialgal strains already deposited at the University of Rostock as well as those from other institutions from around the world were gathered; authentic strains were prioritized when possible. All cultures were maintained at $20-22^{\circ} \mathrm{C}$ on $1.5 \%$ modified Bold's Basal agar (3N BBM + V, Bischoff and Bold 1963, modified by Starr and Zeikus 1993 to have triple nitrate concentration) with a $16: 8$ light: dark photoperiod, with $30 \mu \mathrm{m}$ photons $\mathrm{m}^{-2} \mathrm{~s}^{-1}$ (Lumilux Cool Daylight L18W/840; OSRAM, Munich, Germany).

\section{Qualitative and quantitative LMWC analysis}

Algal suspensions of each strain were grown for four weeks in $150 \mathrm{~mL}$ liquid 3N BBM $+\mathrm{V}$ medium enriched with additional $10 \mathrm{~mL}$ Provasoli's Enrichment Solution per liter, under standard culture conditions outlined above. Medium was refreshed weekly to maintain the algae in the exponential growth phase.

Processing of algal biomass for high-performance liquid chromatography (HPLC) and carbon-13 nuclear magnetic resonance $\left({ }^{13} \mathrm{C}-\mathrm{NMR}\right)$ analysis was done according to Gustavs et al. (2011). HPLC analysis was undertaken on an Agilent 1260 system (Agilent Technologies, Santa Clara, CA, USA) equipped with a differential refractive index detector; one isocratic method was used to verify LMWC concentration and identity within the algal strain extracts. A Phenomenex REZEX ROA-Organic Acid resin-based column with a Phenomenex Carbo-H+ guard cartridge (Phenomenex, Torrance, CA, USA), was used to separate the solutes. The mobile phase consisted of $5 \mathrm{mM} \mathrm{H}_{2} \mathrm{SO}_{4}$ at a flow rate of $0.4 \mathrm{~mL} \mathrm{~min}^{-1}$ at $70^{\circ} \mathrm{C}$.

For ${ }^{13} \mathrm{C}$-NMR spectroscopy, dried algal biomass samples were re-dissolved in $0.5 \mathrm{~mL} \mathrm{D}_{2} \mathrm{O}(99.9 \%)$. The NMR spectra were recorded with a Bruker spectrometer $\left({ }^{1} \mathrm{H}\right.$ : $500.13 \mathrm{MHz} ;{ }^{13} \mathrm{C}$ : $125.8 \mathrm{MHz}$, AVANCE 500 Neo spectrometer (Bruker BioSpin, Ettlingen, Germany). Chemical shifts $\delta$ are given in ppm relative to the signal for internal 


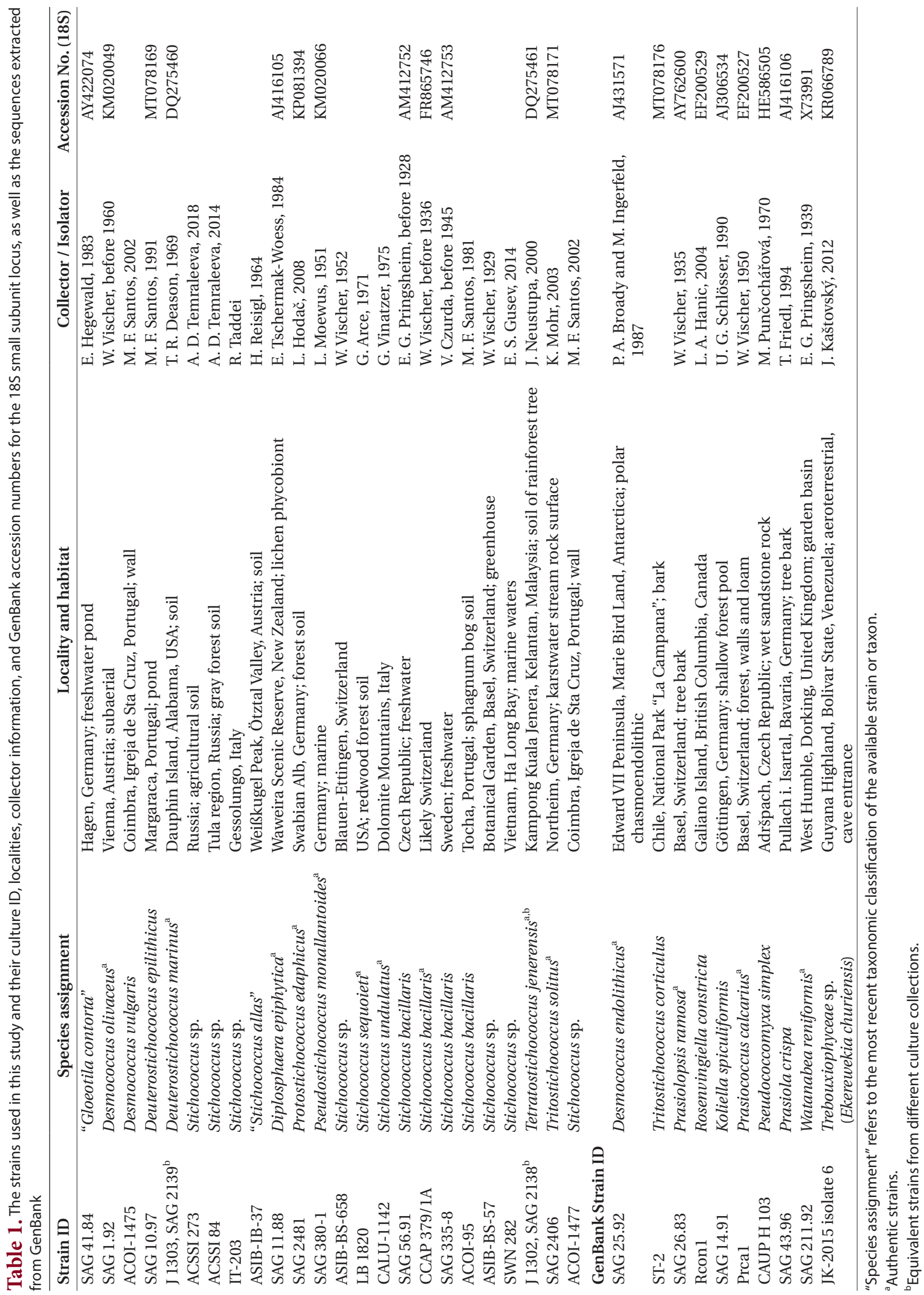


TMS $(\delta=0)$. The calibration of spectra was carried out externally, using the signals of acetone $\left[5 \%, \delta\left({ }^{13} \mathrm{C}\right)=30.3\right.$ $\mathrm{ppm}]$ in $\mathrm{D}_{2} \mathrm{O}$. Samples were run in 5-mm diameter tubes at $298 \mathrm{~K}$. For ${ }^{13} \mathrm{C}$-NMR spectra a sweep width of $30,000 \mathrm{~Hz}$ and a number of 10,000 scans were used.

A battery of common compatible solutes served as standards for interpretation of results. One mM solutions of sorbitol, trehalose mannitol, sucrose, glucose, erythritol, glycerol and ribitol were ran and quantified by peak areas. The limit of detection was $0.01 \mathrm{mM}$ and the limit of quantitation was $0.03 \mathrm{mM}$ for the LMWC standards used. Peak areas in the chromatograms were integrated and correlated to a ten-point calibration curve for each substance detected; resulting concentrations are expressed

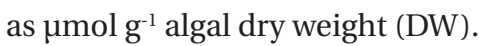

\section{DNA extraction}

Culture material was transferred to $2 \mathrm{~mL}$ tubes. DNA isolation was done with the NucleoSpin Plant II Mini Kit (Macherey and Nagel, Düren, Germany) or Qiagen DNeasy Plant Mini Kit (Qiagen Inc., Hilden, Germany) following their respective product instructions. DNA concentrations were measured using Qubit Fluorometer (ThermoFisher Scientific Germany, Dreiech, Germany). DNA samples were stored at $-18^{\circ} \mathrm{C}$ until further processing.

\section{Polymerase chain reaction and downstream processing}

All polymerase chain reactions (PCRs) amplifying the $18 \mathrm{~S}$ small subunit (SSU) locus were performed with the basic composition of $12.5 \mu \mathrm{L}$ MyTaq Mix (Meridian Bioscience, London, UK), 9.5 $\mu \mathrm{L}$ PCR-grade $\mathrm{H}_{2} \mathrm{O}, 1 \mu \mathrm{L}$ template DNA, and primers $1 \mu \mathrm{L}$ at a concentration of $10 \mathrm{pmol} \mu \mathrm{L}^{-1}$ each. The PCR conditions for each primer pair are outlined in Supplementary Table S1. PCR products were visualized on a 1.5\% agarose gel and cleaned with SureClean Plus (Bioline, Luckenwalde, Germany) following manufacturer's instructions and sequenced by Eurofins Genomics (Ebersberg, Germany) using the same respective PCR primers. All sequences were submitted to the National Center for Biotechnology Information (NCBI); accession numbers for all sequences can be found in Table 1.

The reads were assembled and edited in PhyDE (Müller et al. 2010), automatically aligned using the MUSCLE algorithm (Edgar 2004) with minor manual proofreading. jModelTest (Posada 2008) was used to find the best model for phylogenetic analysis, the result of which was GTR + G + I (Tavaré 1986). The 18 S gene locus was used to obtain phylogenetic trees using the software Geneious v. 8.1.9 (Biomatters Ltd., Auckland, New Zealand) with the add-ons for RAxML (maximum likelihood [ML]) and MrBayes (Bayesian inference $[\mathrm{BI}]$ ), respectively. Both ML and BI analyses used the GTR + G + I model with 4 rate categories, with the $-\mathrm{f}$ a option with 10,000 bootstrap replicates to calculate branch support for the bestscoring tree in ML. This option performs rapid bootstrap analysis and searches for the bestscoring ML tree in one program run. All analyses were conducted under random seed 12,354. The following settings were used for BI: runs with four incrementally heated Metropolis-coupled Monte-Carlo Markov Chains with 5 million generations, burn-in 1,250,000 generations, with a subsampling frequency of 1,000 ; heated chains $=4$, temp. $=0.2$; random seed $=11,244$. The effective sample size value was $>200$ and the trace plot indicated convergence. Introns were ignored for the phylogenetic analyses, since they contributed to artificially high differences in the resulting trees. Phylogenetic trees were edited in FigTree v. 1.4.2 (Rambaut 2008). Gene sequences from several authentic strains were used for the phylogenetic analysis (Table 1). The outgroups were Pseudococcomyxa simplex and Watanabea reniformis.

\section{RESULTS}

\section{Molecular phylogeny}

The 18S SSU phylogeny recovered is mainly congruent with that of the latest study of Stichococcus-like organisms (Pröschold and Darienko 2020, Sommer et al. 2020b), except the clade containing SAG 11.88 Diplosphaera, CAUP J 1302 Tetratostichococcus jenerensis, SAG 2481 Protostichococcus edaphicus, and CAUP J 1303 Deuterostichococcus marinus (Bayesian posterior probability $[\mathrm{B}]=1.00 /$ ML bootstrap = 54) (Fig. 1). The separation between the non-Prasiolaceae and Stichococcus-like organisms was well supported ( $\mathrm{B}=0.99$ / $\mathrm{ML}=79$ ).

The clades Stichococcus bacillaris and Pseudostichococcus segregated with high support $(\mathrm{B}=1.00$ / $\mathrm{ML}=100$ and $\mathrm{B}=1.0 / \mathrm{ML}=99$, respectively). However, there was weak support for the segregation of the genera Deuterostichococcus, Tetratostichococcus, Protostichococcus, and Diplosphaera. Representative strains from each genus were divided into branches or mixed clades. $\mathrm{Deu}$ terostichococcus had particularly bad resolution with a 


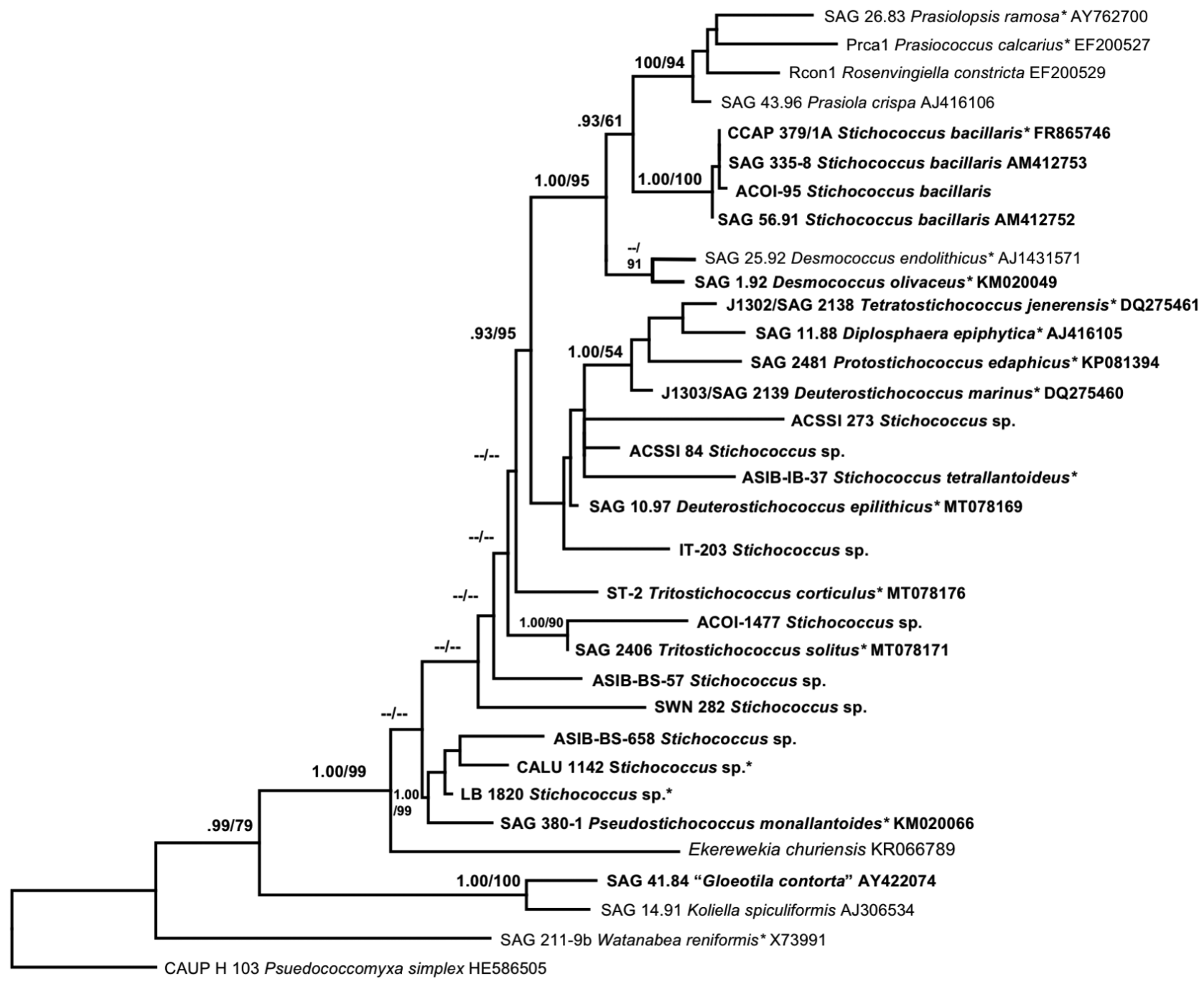

0.05

Fig. 1. 18S small subunit gene tree of the dataset strains. Left branch support values = Bayesian inference $(\mathrm{BI})$, right branch support values = maximum likelihood (ML); BI / ML branch support values over 90/50 are displayed. Bold type indicates strains used in this study. The asterisks (*) indicate authentic strains.

low barcoding gap and contradictory support in the two trees, where the patristic distances ( $p$-distances) ranged from $1.0-3.0 \%$ (BI) and $2.0-9.0 \%$ (ML). The clade containing Pseudostichococcus segregated with high support $(\mathrm{B}=$ $1.00 / \mathrm{ML}=99$ ), apparently with two lineages.

\section{Compatible solute analysis}

The qualitative LMWC production pattern of Stichococcus and Stichococcus-like organisms consist of sorbitol and sucrose (Fig. 2). No other osmolytes were represented in this clade (Fig. 3). NMR analyses verified that the disaccharide of the LMWCs was sucrose and not trehalose, which has a similar retention time in HPLC analysis but differs in molecular structure (Supplementary Fig. S1). There was one strain that had differential osmolyte composition: strain 41.84 Gloeotila contorta lacked sorbitol and produced only sucrose, as well as an unidentified compound. Supplementary Table S2 details the absolute concentrations of sorbitol and sucrose measured in each strain.

Osmolyte concentrations varied widely between the Stichococcus-like strains, ranging from 28 to $\sim 440 \mu \mathrm{mol}$ $\mathrm{g}^{-1} \mathrm{DW}$ for sorbitol and 9 to $\sim 140 \mu \mathrm{mol} \mathrm{g}{ }^{-1} \mathrm{DW}$ for sucrose 


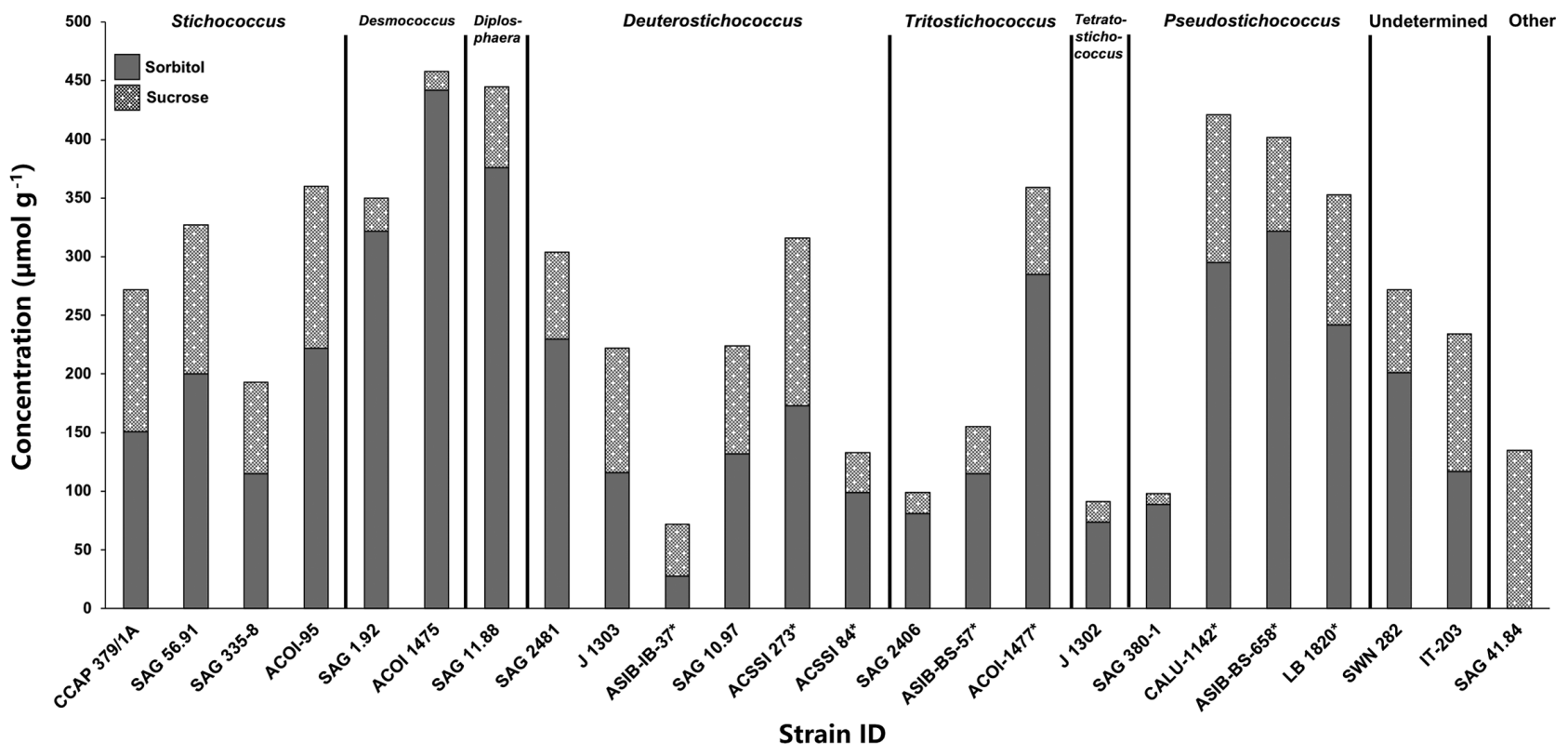

Fig. 2. Proportions of the major osmolytes present in each strain, arranged in the order in which they appear on the phylogenetic tree, separated into their generic assignments (Fig. 1). Unidentified strains did not segregate into a clade in the phylogeny. Concentrations are expressed as $\mu \mathrm{mol} \mathrm{g}^{-1}$ dry weight (DW). The vast majority of strains produced more sorbitol than sucrose, though in some strains within Deuterostichococcus, such as ASIB-IB-37 Deuterostichococcus tetrallantoideus, had an almost $1: 1$ ratio. Strain SAG 41.84 Gloeotila contorta does not belong within the Stichococcus-like clade of the Prasiolales, as it does not produce sorbitol and falls rather into the Koliellaceae. The asterisks $\left(^{*}\right)$ indicate strains putatively belonging into the named genus, based on the preliminary phylogeny.

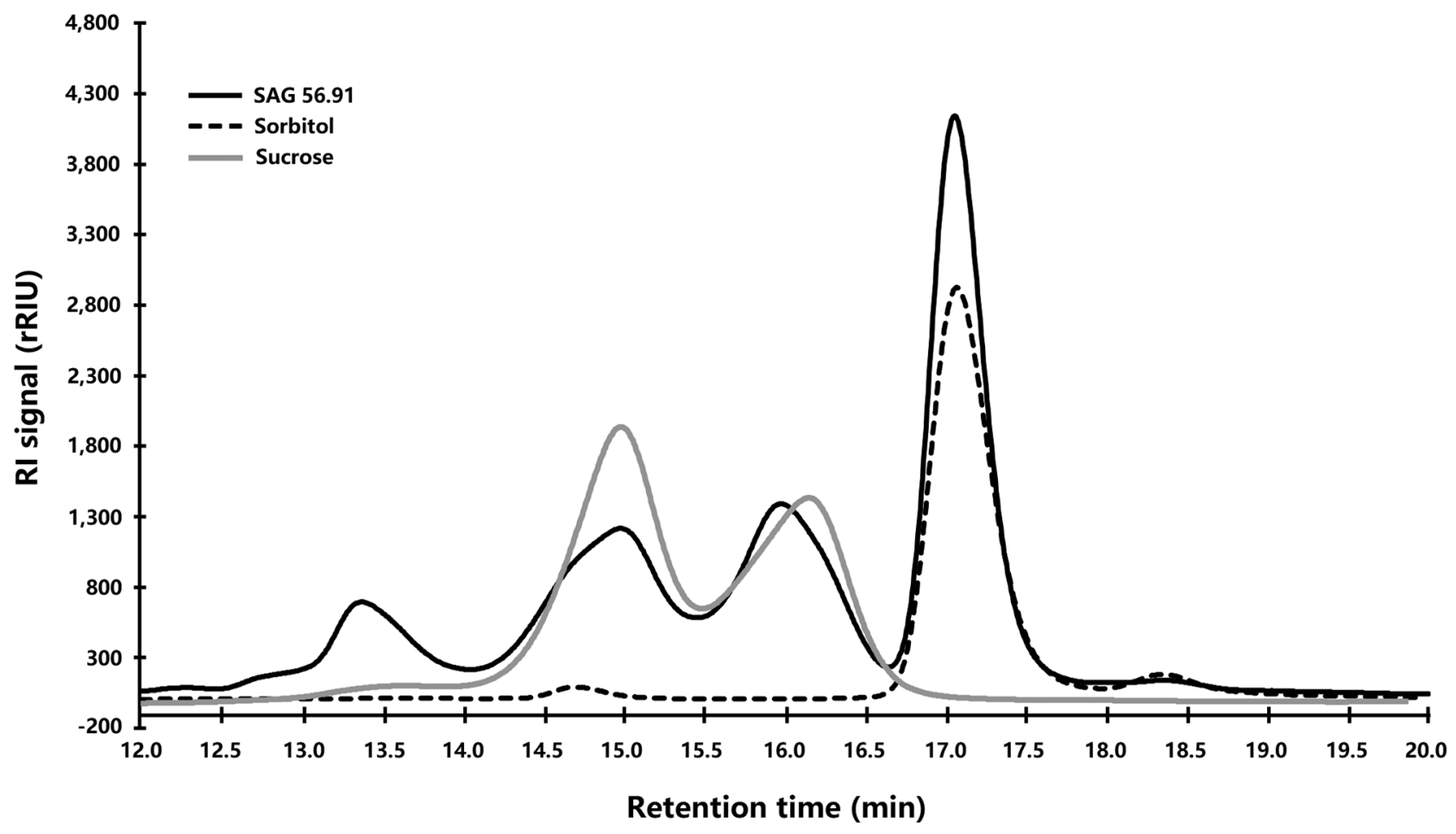

Fig. 3. High-performance liquid chromoatography of cellular extracts of SAG 56.91 Stichococcus bacillaris, which also represents the typical osmolyte expression pattern of Stichococcus-like strains. Sorbitol shows a peak at $17.1 \mathrm{~min}$, sucrose at 15.1 and $16.3 \mathrm{~min}$. RI signal, refractive index signal; rRIU, relative refractive index units. 
(Fig. 2). Desmococcus strains SAG 1.92 and ACOI-1475 had the highest concentrations of sorbitol at 322 and 441 $\mu$ mol g ${ }^{-1} \mathrm{DW}$, respectively; the lowest sorbitol concentration well under $100 \mu \mathrm{mol} \mathrm{g}^{-1} \mathrm{DW}$ were detected in the strains ASIB-IB-37 Deuterostichococcus tetrallantoideus, CAUP J 1302 T. jenerensis, SAG 2406 Tritostichococcus solitus, and SAG 380-1 Pseudostichococcus monallantoides (Fig. 2). As groups, Desmococcus, Diplosphaera and the strains putatively belonging to Pseudostichococcus had the highest sorbitol production.

The ratio of sorbitol : sucrose varied between the strains and there was little strongly genus-specific ratio correlations to be seen. The $S$. bacillaris group was fairly consistent in this ratio, ranging from 1.2-1.6. The two Desmococcus strains had remarkably high ratios of 11.5 $: 1$ and $27.6: 1$.

\section{DISCUSSION}

The strains historically identified as Stichococcus have very similar morphology, which, through genetic techniques of recent years, have been shown to have a higher-than-expected diversity. The thorough investigation by Pröschold and Darienko (2020) indicated that the morphologies of Stichococcus, Protostichococcus, Deuterostichococcus, Tritostichococcus, Teratostichococcus, and Pseudostichococcus are, in most cases, both extremely variable within infrageneric taxa yet similar between different genera. Their separation and subsequent description of the genera resulted in there being virtually no traditional autapomorphies, such as differences in cell shape, length : width ratio, chloroplast form, and pyrenoid presence within each genus. The basic descriptive traits of the genera were "cylindrical, hyaline, chloroplast parietal and unlobed, without mucilaginous layer, with or without pyrenoid," with cell dimensions as a main character. However, quantification of the length : width ratio shows that only Diplosphaera can be distinguished from the other Stichococcus-like genera (Pröschold and Darienko 2020, fig. 14). Only Diplosphaera and the cluster-forming Desmococcus can be reliably separated from the other Stichococcus-like genera on the basis of morphology. It is reasonable to say that for morphological identification purposes from environmental data, most Stichocococcus-like algae are pseudocryptic. Likewise, the literature dedicated to characterizing these new strains is hitherto scare. The role of chemotaxonomic analysis here was to bolster the existing knowledge on non-morphological traits of these newly described strains, since the morphological descriptions are vague and very little is known about their physiology.

The phylogeny in this study was constructed solely to orient results and not to provide a thorough investigation into the phylogenetic relationships between the taxa. Furthermore, it is oriented to-and with sequences from - the phylogeny from Pröschold and Darienko (2020), as these authors redefined the seven Stichococcus-like lineages. Thus, we chose the most widely accepted locus for green algal phylogenetic analysis, the $18 \mathrm{~S}$ SSU locus and chose to disregard the single Group 2 intron within the locus. Even without using an exhaustive marker array, the overall pattern of clade segregation is consistent with the recently established lineages. Another independent study has also done this in a different context and acquired similar results (Sommer et al. 2020b). Several "Stichococcus sp." strains tested this phylogenetic hypothesis as well as provided material for the chemosystematic comparison.

The taxon S. bacillaris Nägeli 1849 is currently one of the few within Stichococcus s.l. confirmed to be of this genus, as many others, such as S. jenerensis and S. allas, have been transferred to other Stichococcus-like clades or genera. In this study's phylogeny, the $S$. bacillaris clade's separation and close relationship to Desmococcus parallel the results from the two newest phylogenetic studies. It was not possible to obtain high-quality $18 \mathrm{~S}$ sequences from strain ACOI-1475 Desmococcus and, thus, was subsequently excluded from the phylogeny, but its chemotaxonomy and morphology were consistent with that of the generitype strain SAG 1.92 Desmococcus olivaceus. Protostichococcus, Diplosphaera, and Tetratostichococcus, as sorbitol and sucrose producers, align with other members of the Prasiola-clade and are genetically distinct from Stichococcus s.s. and Pseudostichococcus.

Type material for S. allas Reisigl 1964, a taxon that now falls within Deuterostichococcus after the latest revision, was rediscovered by the authors of the present study and examined chemotaxonomically, with ramifications for the taxonomy of the clade. This strain, ASIB-IB-37, was assumed to be unavailable and hence not directly described or assigned in Pröschold and Darienko (2020), which means that the reclassification of S. allas into Deuterostichococcus allas was necessarily preliminary, pending type material analysis. In an amendment by the same authors, the taxon S. allas was subsequently declared a heterotypic synonym of Chodatia tetrallantoidea Kol 1934 (morphologically identical to S. allas) and D. allas and was then reassigned to D. tetrallantoideus (Pröschold et al. 2020). After comparing the genetic sequences of $S$. 

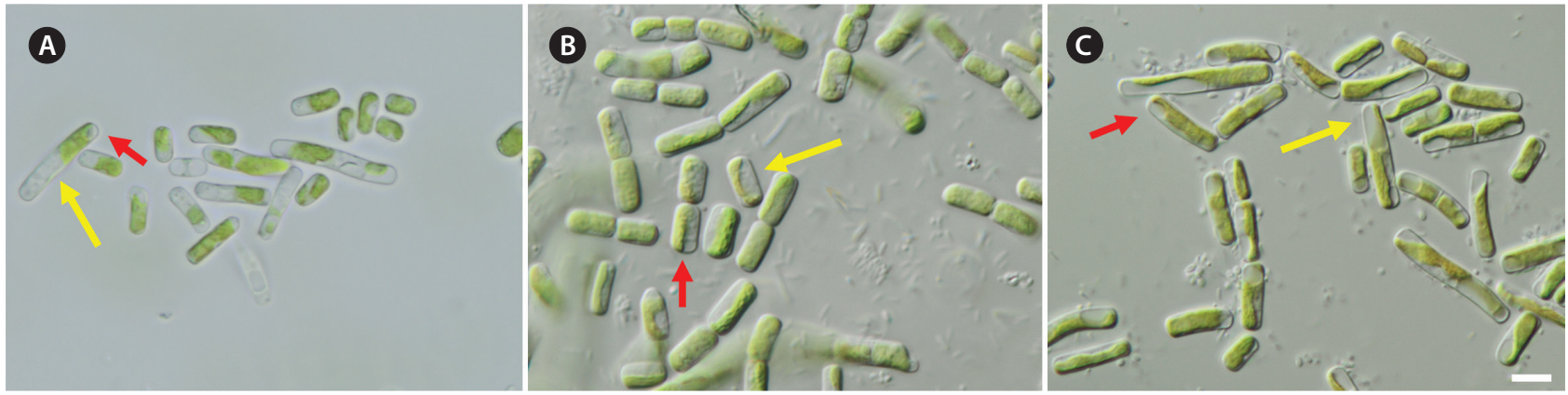

Fig. 4. Morphologies of SAG 380-1 Pseudostichococcus monallantoides (A), CALU 1142 Stichococcus undulatus (B), and LB 1820 Stichococcus sequoieti (C). Yellow arrows emphasize chloroplast form, red arrows the cell habitus. In SAG 380-1 and LB 1820, filaments are rarely seen, while in CALU 1142 short filaments are typical. The chloroplasts cover half the cell length in SAG 380-1, CALU 1142 vertically, and almost the entire cell horizontally in LB 1820. All three strains are characterized by relatively high length : width ratios (although SAG 380-1 has the smallest cells), a cylindrical cell shape with a squared-off appearance at the ends, and frequently large oil and starch droplets in the cells. Scale bar represents: $5 \mu \mathrm{m}$. [Colour figure can be viewed at http://www.e-algae.org].

allas and D. tetrallantoideus as well as the osmolyte production pattern of strain ASIB-IB-37, it seems that the type strain of "S. allas" falls within the near vicinity of Deuterostichococcus. Thus, the taxonomic reassignment of strain ASIB-IB-37 be the heterotypic type material of $D$. tetrallantoideus is valid and supports the revision in Pröschold and Darienko (2020).

However, there seems to be insufficient support in the 18 locus to establish Deuterostichococcus as a monophyletic clade, especially when including sequences from unidentified strains. In particular, the supports for the branches containing ASIB-IB-37, SAG 10.97, and J 1303 are below the support threshold. A polytomy occurs between the strains ACSSI-84, ASIB-IB-37, and SAG 10.97. Morphological data also does not improve resolution. Of the unidentified strains only ACSSI-84 exhibits a pyrenoid which fits the classical description of $S$. deasonii (D. marinus), but this trait no longer supports an autapomorphy (Pröschold and Darienko 2020). To resolve this clade would go beyond the scope of this paper; for now, there is not enough evidence to reassign these three strains into Deuterostichococcus.

Assigning strain ACOI-1477 to Tritostichococcus is somewhat easier, although still not entirely clear. ACOI1477 only has five base-pair differences to SAG 2406 ( $p$ distance $=1.0 \%$ BI, 3.0\% ML). Problematic is strain ST-2, which does not segregate with the clade containing the other Tritostichococcus strain. Morphologically, ACOI1477 could fit into either Tritostichococcus or Deuterostichococcus, as it cylindrical, has a single smooth parietal chloroplast without pyrenoid, and has a length : width ratio of $\sim 2$.

In Sommer et al. (2020b), one of the few works that have identified Stichococcus-like organisms from environmental samples on the basis of molecular data, it was shown that Pseudostichococcus consists of two separate clades. Our phylogeny bolsters this finding, as the strains ASIB-BS-658, CALU 1142, and LB 1820 form a sister clade to SAG 380-1 P. monallantoides $(\mathrm{BI}=1.00, \mathrm{ML}=99)$. Although this is not definitive, it supports the conclusion that these three strains are more related to Pseudostichococcus than to the other Stichococcus-like strains. In addition, all these follow the Stichococcus-like sorbitol and sucrose production pattern. However, the taxonomic history of these three taxa has uncertainties that we attempt to clarify here.

To examine the validity of this transfer into Pseudostichococcus Moewus 1951, it was necessary to analyze the strains CALU 1142 S. undulatus and LB 1820 S. sequoieti, the authentic strains of their respective taxa (Fig. 4). Unfortunately, there has not yet been a direct comparison of their differences to one another, although the morphological similarity between these two strains has been noticed indirectly in the past by both authorities Vinatzer (1975) and Arce (1971), respectively. S. undulatus has a similar size range $(w 4 \mu \mathrm{m} ; l 5-30 \mu \mathrm{m})$ to $S$. sequoieti ( $w$ $1.5-3 \mu \mathrm{m} ; l 5-25 \mu \mathrm{m})$, the latter slightly smaller in both dimensions. Vinatzer wrote explicitly in his description of $S$. undulatus that $S$. mirabilis is similar to S. undulatus, except that $S$. mirabilis does not form filaments. Furthermore, the illustrations from Vinatzer indicate that $S$. undulatus has a chloroplast that fills the cell length, but only halfway in height. In the original description of $S$. sequoieti, Arce (1971) compared S. sequoieti against several other Stichococcus strains, one of which was S. mirabilis Lagerheim (strain 316) from the Indiana Univer- 
Van et al. Osmolyte Taxonomy in Stichococcus Genera

\begin{tabular}{l|l|ll}
\cline { 2 - 3 } TTGGAT & TGGCGGCAGGGGGCGG & TTTCCG & sAg 380-1 Pseudostichococcus monallantoides \\
TTGGAT & TGGCGGCAGGGGGCGG & TTT-CC & CALU-1142 Stichococcus undulatus \\
TTGGAT & TGGCGGCAGGGGGCGG & TTTCCG & LB 1820 Stichococcus sequoieti \\
TTGGAT & TGGCGGCAGGGGGCGG & TTTCCG & ASIB-BS-658 Stichococcus sp. \\
TTGGAT & CGGCGACAGCCGGCGG & TAACGC & SAG 2138 Tetratostichococcus jenerensis \\
TTGGAT & TGGCGATTGTCGGCGG & AAACSS & CAP 379/1A Stichococcus bacillaris \\
TTGGAT & TGGCGACTTTCGGCGG & AACGCC & SAG 2481 Protostichococcus edaphicus
\end{tabular}

Fig. 5. Nucleotide sequence schematic of the $18 \mathrm{~S}$ small subunit rDNA V9 region Helix 49 (boxed), diagnostic for the genus Pseudostichococcus. For comparison are three authentic strains from other Stichococcus-like genera that differ from Pseudostichococcus.

sity Culture Collection of Algae (IUCC, now UTEX), with the conclusion that $S$. sequoieti differs from $S$. mirabilis in dimensions ( $w 2.3-3.5 \mu \mathrm{m} ; l 30 \mu \mathrm{m})$ and that chloroplast length in $S$. mirabilis reaches $1 / 2$ to $1 / 3$ cell length whereas that of S. sequoieti fills the cell. Starch and lipids are absent in both. Unfortunately, strain UICC 316 is unavailable, as is the only other known strain of S. mirabilis (CCAP 379/3), so it was not possible to compare S. mirabilis to S. sequoieti and S. undulatus directly. Here we take S. undulatus over S. mirabilis as the strain identity and point of comparison to $S$. sequoieti due to type material availability and due to the fact that $S$. undulatus forms short filaments in culture (Fig. 4). The 11/1,760 base pairs difference $(p$-distance $=1.0 \% \mathrm{BI} ; 3.0 \% \mathrm{ML}$ ) in the $18 \mathrm{~S}$ sequence strongly suggests that $S$. undulatus and S. sequoieti are distinct taxa; for reference, S. sequoieti-P. monallantoides $p$-distance $=1.0 \% \mathrm{BI} ; 2.0 \% \mathrm{ML}$; . undulatus $-P$. monallantoides $=2.0 \%$ BI; $5.0 \%$ ML. Furthermore, the diagnostic 18S SSU rDNA V9 Helix 49 region (Fig. 5) for Pseudostichococcus is identical in all four strains. In this sense, we partially agree with Pröschold and Darienko (2020) that S. sequoieti does not belong in Stichococcus s.s.; however, it does belong within the Stichococcus-like group. In light of the physiological and phylogenetic results, these two strains could be transferred to the hitherto monotypic genus Pseudostichococcus. Strain ASIB-BS-658 has the same V9 Helix 49 region, as well as sequence similarity, to the other Pseudostichococcus strains and should also be placed into this genus.

Apart from verifying clade membership, we were able to conclude based on the osmolyte composition that SAG 41.84 does not belong to the Stichococcus-like organisms due to its conspicuous lack of sorbitol. Our chemotaxo- nomic results support the conclusions of a previous phylogenetic analysis (Henley et al. 2004), which showed that strain SAG 41.84 does not fit into either the Picochlorum / Nannochloris-like clade, nor Stichococcus s.l., nor Gloeotila s.l. Following the reassignment of many members of the genus Gloeotila by Pröschold and Darienko (2020) to S. bacillaris, it appears that this strain rather belongs to another family of the order Prasiolales, most likely within the Koliellaceae. However, it is not possible to precisely assign this taxon into an existing genus without further taxonomic and nomenclatural work in both Koliella and the historical Gloeotila clade, barring more precise investigation into the type of the name of the genus, Gloeotila oscillarina Kützing. "Gloeotila contorta" SAG 41.84 also does not produce osmolytes typical of other closely related groups, such as ribitol or glycerol (Chloroidium, Watanabea, Viridiella, from Darienko et al. 2010), or erythritol (Apatococcus, from Gustavs et al. 2011).

The results from the LMWC analysis from this study are in agreement with those of other studies on Trebouxiophyceaen algae (Brown 1977, Brown and Hellebust 1980, Gustavs et al. 2011, Hotter et al. 2018), where it was shown repeatedly that members of the Prasiola clade produce sorbitol as an osmolyte. In particular, Brown (1977) and Brown and Hellebust (1978) studied the compatible solute composition of $S$. bacillaris strains directly and showed that sorbitol is the main carbohydrate produced, with proline as an additional osmoprotectant. Our results show that this is a stable (chemo) taxonomic character within the Stichococcus-like genera. Sucrose was also present in all Stichococcus-like strains, but since it is present in many other green algal families, it is not necessarily taxonomically indicative (Brown and Helle- 
bust 1980, Darienko et al. 2015). However, the combination of sorbitol + sucrose may be consistent within the genera studied. A recent work by Medwed et al. (2021) also shows that Diplosphaera produces sorbitol + sucrose during growth. These findings support the phylogenetic exclusion of the algal strain SAG 48.41 from the Stichococcus-like genera, as it does not produce sorbitol.

Finally, the quantitative data obtained on sorbitol and sucrose production in the Stichococcus-like strains provide no phylogenetic resolution. Although there is a slight pattern in the sorbitol : sucrose ratios to be seen (Fig. 2), it is not significant when controlled for genus relationships. Sorbitol is a superior compatible solute compared to sucrose and does not interfere with cellular processes even at high intracellular concentrations (Roberts 2005). The main function of sucrose is, in addition, not that of an osmolyte but rather as a storage compound, as it is the main product of photosynthesis (Everard and Loescher 2016), and hence it only partially contributes to osmotic acclimation. The lower levels of sucrose production in comparison to sorbitol in almost all strains studied support this.

It is unclear why the Desmococcus and Pseudostichococcus, as well as SAG 11.88 Diplosphaera epiphytica, have a higher sorbitol : sucrose ratio than the other Stichococcus-like groups. All strains were cultivated under identical conditions, so that the measured concentrations reflect the specific phenotypic biosynthesis. More empirical data, possibly from different cultivation and experimental conditions, would be necessary to determine a relationship between the sorbitol : sucrose ratio and osmotic stress tolerance. This is particularly relevant since the Stichococcus-like genera are common aeroterrestrial algae and lichen symbionts (Thüs et al. 2011, Fontaine et al. 2013), and it would be expected that they perform similarly under desiccation.

In conclusion, Stichococcus-like algae produce sorbitol and sucrose that serve as compatible solutes to counteract osmotic stress, mainly due to frequent desiccation in the terrestrial habitat. This is a chemotaxonomic marker consistent within the Prasiola clade in the Trebouxiophyceae. The novel results show that the newly erected genera formerly in Stichococcus sensu lato (Protostichococcus, Deuterostichococcus, Tritostichococcus, Tetratostichococcus as well as Pseudostichococcus) share this trait and fall phylogenetically into the Prasiolales, although osmolyte analysis alone is insufficient to delineate their generic boundaries. A taxonomically and historically related "Gloeotila" strain was reassigned to Trebouxiophyceae incertae ordinis based on differential compatible solute production as well as phylogenetic distance. Furthermore, we propose that the taxa Stichococcus undulatus and Stichococcus sequoieti be reassigned into the hitherto monotypic genus Pseudostichococcus.

\section{Summary of proposed taxonomical changes}

Because many of the strains in this study are currently known in the respective culture collections as S. bacillaris or even solely as Stichococcus sp., it will be necessary to reassign them formally into the newly proposed genera of the Stichococcus-like clades. Based on the compatible solute analysis, it is possible to confirm or exclude membership from this broad group. Combined with the preliminary $18 \mathrm{~S}$ phylogeny, this should be sufficient to reassign the following taxa into their new genera. Precise genus-level assignment for the taxa known only generically will require more detailed phylogenetic analysis using the full 18S-28S SSU locus, ideally in combination with plastid markers such as $r b c$ L. However, taxa with a valid botanical name are easily transferred, as they serve as their own basionyms and their original descriptions are still valid. The following new combinations are proposed:

Pseudostichococcus sequoieti (G. Arce) Van \& Glaser, comb. nov.

Basionym: Stichococcus sequoieti G. Arce (1971:25, figs 1-16).

Designated type strain: Strain LB 1820, UTEX Culture Collection of Algae, University of Texas at Austin, Texas, USA.

Pseudostichococcus undulatus (Vinatzer) Van \& Glaser, comb. nov.

Basionym: Stichococcus undulatus Vinatzer (1975, p. 203, fig. 10).

Designated type strain: CALU 1142 Stichococcus undulatus, CALU St. Petersburg Culture Collection, St. Petersburg, Russia.

Sister strain: ASIB-V164.

\section{ACKNOWLEDGEMENTS}

This cooperation would not have been possible without the generous materials support from Dr. Anna Temraleeva (Algal Collection of Soil Science Institute, Puschchino, Russia), Dr. Pavel Škaloud (Charles University, Prague, Czech Republic), Dr. Lira Gaysina (Resource Centre Culture Collection of Microorganisms, St. Petersburg, 
Russia), Dr. Tatiana Mikhailyuk (M.K. Kholodny Institute of Botany, Kiev, Ukraine) and Dr. Andreas Holzinger (University of Innsbruck, Innsbruck, Austria). Many thanks in particular to Dr. Rhena Schumann and Dr. Dirk Michalik at the University of Rostock for guidance in HPLC and NMR methodology. Within the framework of the German Research Foundation Special Priority Project: 1991 Taxon-Omics (CrustAlgaeTax-GL909/1-1), we were able to offer additional and critical data on the taxonomic revision of this genus using a chemotaxonomic approach.

\section{CONFLICTS OF INTEREST}

The authors declare that they have no potential conflicts of interest.

\section{SUPPLEMENTARY MATERIALS}

Supplementary Table S1. A list of primers for nuclear 18 small subunit gene locus and their references (https:// www.e-algae.org).

Supplementary Table S2. Absolute concentrations of sorbitol and sucrose measured in cellular extracts of the strains during high-performance liquid chromatography analysis (https://www.e-algae.org).

Supplementary Fig. S1. $\mathrm{C}^{13}$-NMR spectrum of sugar alcohols produced by SAG 56.91 Stichococcus bacillaris, overlaid with spectra for sorbitol and sucrose (https:// www.e-algae.org).

\section{REFERENCES}

Arce, G. 1971. Stichococcus sequoieti sp. nov. In Parker, B. C. \& Brown, R. M. (Eds.) Contributions in Phycology. Allen Press, Lawrence, KS, pp. 25-30.

Brown, L. M. 1977. Osmoregulatory mechanisms of a euryhaline alga, Stichococcus bacillaris (Chlorophyceae). J. Phycol. 13:9.

Brown, L. M. \& Hellebust, J. A. 1978. Sorbitol and proline as intracellular osmotic solutes in the green alga Stichococcus bacillaris. Can. J. Bot. 56:676-679.

Brown, L. M. \& Hellebust, J. A. 1980. Some new taxonomic characteristics applied to Stichococcus bacillaris (Chlorophyceae). Can. J. Bot. 58:1405-1411.

Darienko, T., Gustavs, L., Eggert, A., Wolf, W. \& Pröschold, T. 2015. Evaluating the species boundaries of green microalgae (Coccomyxa, Trebouxiophyceae, Chlorophyta) using integrative taxonomy and DNA barcoding with further implications for the species identification in environmental samples. PLoS ONE 10:e0127838.

Darienko, T., Gustavs, L., Mudimu, O., Menendez, C. R., Schumann, R., Karsten, U., Friedl, T. \& Pröschold, T. 2010. Chloroidium, a common terrestrial coccoid green alga previously assigned to Chlorella (Trebouxiophyceae, Chlorophyta). Eur. J. Phycol. 45:79-95.

Edgar, R. C. 2004. MUSCLE: multiple sequence alignment with high accuracy and high throughput. Nucleic Acids Res. 32:1792-1797.

Eggert, A. \& Karsten, U. 2010. Low molecular weight carbohydrates in red algae-an ecophysiological and biochemical perspective. In Red algae in the genomic age. Springer, Dordrecht, pp. 443-456.

Everard, J. D. \& Loescher, W. H. 2016. Primary products of photosynthesis, sucrose and other soluble carbohydrates. In Thomas, B., Murray, B. G. \& Murphy, D. J. (Eds.) Encyclopedia of Applied Plant Sciences. Academic Press, Waltmam, MA, pp. 96-104.

Ferrenberg, S., Tucker, C. L. \& Reed, S. C. 2017. Biological soil crusts: diminutive communities of potential global importance. Front. Ecol. Environ. 15:160-167.

Fontaine, K. M., Stocker-Wöergöetter, E., Booth, T. \& PierceyNormore, M. D. 2013. Genetic diversity of the lichenforming alga, Diplosphaera chodatii, in North America and Europe. Lichenologist 45:799-813.

Glaser, K., Baumann, K., Leinweber, P., Mikhailyuk, T. \& Karsten, U. 2018. Algal richness in BSCs in forests under different management intensity with some implications for P cycling. Biogeosciences 15:4181-4192.

Guiry, M. 2012. How many species of algae are there? J. Phycol. 48:1057-1063.

Gustavs, L., Görs, M. \& Karsten, U. 2011. Polyol patterns in biofilm-forming aeroterrestrial green algae (Trebouxiophyceae, Chlorophyta). J. Phycol. 47:533-537.

Hallmann, C., Stannek, L., Fritzlar, D., Hause-Reitner, D., Friedl, T. \& Hoppert, M. 2013. Molecular diversity of phototrophic biofilms on building stone. FEMS Microbiol. Ecol. 84:355-372.

Handa, S., Nakahara, M., Tsubota, H., Deguchi, H. \& Nakano, T. 2003. A new aerial alga, Stichococcus ampulliformis sp. nov. (Trebouxiophyceae, Chlorophyta) from Japan. Phycol. Res. 51:203-210.

Hellebust, J. A. 1985. Mechanisms of response to salinity in halotolerant microalgae. In Pasternak, D. \& San Pietro, A. (Eds.) Biosalinity in Action: Bioproduction with Saline Water. Springer, Dordrecht, pp. 69-81.

Henley, W. J., Hironaka, J. L., Guillou, L., Buchheim, M. A., Buchheim, J. A., Fawley, M. W. \& Fawley, K. P. 2004. 
Phylogenetic analysis of the 'Nannochloris-like' algae and diagnoses of Picochlorum oklahomensis gen. et sp. nov. (Trebouxiophyceae, Chlorophyta). Phycologia 43:641-652.

Hodač, L., Hallmann, C., Spitzer, K., Elster, J., Faßhauer, F., Brinkmann, N., Lepka, D., Diwan, V. \& Friedl, T. 2016. Widespread green algae Chlorella and Stichococcus exhibit polar-temperate and tropical-temperate biogeography. FEMS Microbiol. Ecol. 92:fiw122.

Holzinger, A. \& Karsten, U. 2013. Desiccation stress and tolerance in green algae: consequences for ultrastructure, physiological and molecular mechanisms. Front. Plant Sci. 4:327.

Holzinger, A. \& Pichrtová, M. 2016. Abiotic stress tolerance of charophyte green algae: new challenges for omics techniques. Front. Plant Sci. 7:678.

Hotter, V., Glaser, K., Hartmann, A., Ganzera, M. \& Karsten, U. 2018. Polyols and UV-sunscreens in the Prasiolaclade (Trebouxiophyceae, Chlorophyta) as metabolites for stress response and chemotaxonomy. J. Phycol. 54:264-274.

Kamiya, M., West, J. A., Karsten, U. \& Ganesan, E. K. 2016. Molecular and morphological delineation of Caloglossa beccarii and related species (Delesseriaceae, Rhodophyta). Phycologia 55:640-649.

Karsten, U., Friedl, T., Schumann, R., Hoyer, K. \& Lembcke, S. 2005. Mycosporine-like amino acids and phylogenies in green algae: Prasiola and its relatives from the Trebouxiophyceae (Chlorophyta). J. Phycol. 41:557-566.

Karsten, U., Görs, S., Eggert, A. \& West, J. A. 2007. Trehalose, digeneaside, and floridoside in the Florideophyceae (Rhodophyta): a reevaluation of its chemotaxonomic value. Phycologia 46:143-150.

Karsten, U., Herburger, K. \& Holzinger, A. 2016. Living in biological soil crust communities of African deserts: physiological traits of green algal Klebsormidium species (Streptophyta) to cope with desiccation, light and temperature gradients. J. Plant Physiol. 194:2-12.

Karsten, U., West, J. A., Zuccarello, G. C., Nixdorf, O., Barrow, K. D. \& King, R. J. 1999. Low molecular weight carbohydrate patterns in the Bangiophyceae (Rhodophyta). J. Phycol. 35:967-976.

Kitzing, C., Pröschold, T. \& Karsten, U. 2014. UV-induced effects on growth, photosynthetic performance and sunscreen contents in different populations of the green alga Klebsormidium fluitans (Streptophyta) from alpine soil crusts. Microb. Ecol. 67:327-340.

Medwed, C., Holzinger, A., Hofer, S., Hartmann, A., Michalik, D., Glaser, K. \& Karsten, U. 2021. Ecophysiological, morphological, and biochemical traits of free-living
Diplosphaera chodatii (Trebouxiophyceae) reveal adaptation to harsh environmental conditions. Protoplasma Advanced online publication. https://doi.org/10.1007/ s00709-021-01620-6.

Moewus, L. 1951. Systematische Bestimmung einzelliger grüner Algen auf Grund von Kulturversuchen (Sphaerosorus composita, Oocystis marina und Pseudostichococcus monallantoides). Bot. Not. 4:287-318.

Müller, K., Müller, J., Neinhuis, C. \& Quandt, D. 2010. PhyDE: Phylogenetic data editor, version 0.9971. Available from: http://www. phyde.de. Accessed May 30, 2021.

Mutaf, T., Oz, Y., Kose, A., Elibol, M. \& Oncel, S. S. 2019. The effect of medium and light wavelength towards Stichococcus bacillaris fatty acid production and composition. Bioresour. Technol. 289:121732.

Neustupa, J., Eliáš, M. \& Šejnohová, L. 2007. A taxonomic study of two Stichococcus species (Trebouxiophyceae, Chlorophyta) with a starch-enveloped pyrenoid. Nova Hedwigia 84:51-63.

Neustupa, J. \& Škaloud, P. 2008. Diversity of subaerial algae and cyanobacteria on tree bark in tropical mountain habitats. Biologia 63:806-812.

Neustupa, J. \& Škaloud, P. 2010. Diversity of subaerial algae and cyanobacteria growing on bark and wood in the lowland tropical forests of Singapore. Plant Ecol. Evol. 143:51-62.

Olivieri, G., Marzocchella, A., Andreozzi, R., Pinto, G. \& Pollio, A. 2011. Biodiesel production from Stichococcus strains at laboratory scale. J. Chem. Technol. Biotechnol. 86:776-783.

Oren, A. \& Gunde-Cimerman, N. 2007. Mycosporines and mycosporine-like amino acids: UV protectants or multipurpose secondary metabolites? FEMS Microbiol. Lett. 269:1-10

Orfanoudaki, M., Hartmann, A., Ngoc, H. N., Gelbrich, T., West, J., Karsten, U. \& Ganzera, M. 2020. Mycosporinelike amino acids, brominated and sulphated phenols: suitable chemotaxonomic markers for the reassessment of classification of Bostrychia calliptera (Ceramiales, Rhodophyta). Phytochemistry 174:112344.

Pollio, A., Aliotta, G., Pinto, G., Paterno, M. \& Bevilacqua, A. 1997. Ecophysiological characters and biochemical composition of Stichococcus bacillaris Naegeli strains from low pH environments. Algol. Stud. 84:129-143.

Posada, D. 2008. jModelTest: phylogenetic model averaging. Mol. Biol. Evol. 25:1253-1256.

Pröschold, T. \& Darienko, T. 2020. The green puzzle Stichococcus (Trebouxiophyceae, Chlorophyta): new generic and species concept among this widely distributed genus. Phytotaxa 441:113-142. 
Pröschold, T., Darienko, T. \& Guiry, M. D. 2020. Nomenclatural corrections in the green algal genus Deuterostichococcus Pröschold \& Darienko (Trebouxiophyceae). Notulae Algarum 137:1-2.

Rambaut, A. 2008. Figtree version 1.2.2. Institute of Evolutionary Biology, University of Edinburgh. Available from: http://tree.bio.ed.ac.uk/software/figtree. Accessed May $30,2021$.

Rindi, F. 2007. Diversity, distribution and ecology of green algae and cyanobacteria in urban habitats. In Seckbach, J. (Ed.) Algae and Cyanobacteria in Extreme Environments: Cellular Origina, Life in Extreme Habitats and Astrobiology. Springer, Dordrecht, pp. 619-638.

Roberts, M. F. 2005. Organic compatible solutes of halotolerant and halophilic microorganisms. Saline Syst. 1:5.

Sommer, V., Karsten, U. \& Glaser, K. 2020a. Halophilic algal communities in biological soil crusts isolated from potash tailings pile areas. Front. Ecol. Evol. 8:46.

Sommer, V., Mikhailyuk, T., Glaser, K. \& Karsten, U. $2020 b$. Uncovering unique green algae and cyanobacteria isolated from biocrusts in highly haline potash tailing pile habitats using an integrative approach. Microorganisms
8:1667.

Starr, R. C. \& Zeikus, J. A. 1993. Utex: the culture collection of algae at The University of Texas at Austin 1993 list of cultures. J. Phycol. 29:1-106.

Tavaré, S. 1986. Some probabilistic and statistical problems in the analysis of DNA sequences. In Miura, R. (Ed.) Some Mathematical Questions in Biology: DNA Sequence Analysis. Vol. 17. Lectures on Mathematics in the Life Sciences. American Mathematical Society, Providence, RI, pp. 57-86.

Thüs, H., Muggia, L., Pérez-Ortega, S., Favero-Longo, S. E., Joneson, S., O’Brien, H., Nelsen, M. P., Duque-Thüs, R., Grube, M., Friedl, T., Brodie, J., Andrew, C. J., Lücking, R., Lutzoni, F. \& Gueidan, C. 2011. Revisiting photobiont diversity in the lichen family Verrucariaceae (Ascomycota). Eur. J. Phycol. 46:399-415.

Uher, B. 2008. Spatial distribution of cyanobacteria and algae from the tombstone in a historic cemetery in Bratislava, Slovakia. Fottea 9:81-92.

Vinatzer, G. 1975. Neue Bodenalgen aus den Dolomiten. Plant Syst. Evol. 123:213-235. 